\title{
Thermal Transport in Oxidized Polycrystalline Graphene
}

Xin $\mathrm{Mu}^{1}$, Zhigong Song ${ }^{2}$, Yanlei Wang ${ }^{2}$, Zhiping $\mathrm{Xu}^{2}$, David B. Go ${ }^{1,3}$ and Tengfei Luo ${ }^{1,4^{*}}$

${ }^{1}$ Department of Aerospace and Mechanical Engineering, University of Notre Dame, Notre Dame, IN 46556,USA

${ }^{2}$ Applied Mechanics Laboratory, Department of Engineering Mechanics, and Center for Nano and Micro Mechanics, Tsinghua University, Beijing 100084, People's Republic of China

${ }^{3}$ Department of Chemical and Biomolecular Engineering, University of Notre Dame, Notre Dame, IN 46556, USA

${ }^{4}$ Center for Sustainable Energy at Notre Dame, University of Notre Dame, Notre Dame, IN 46556, USA

*Corresponding author. Tel: 1-574-631-9683. E-mail: tluo@nd.edu (Tengfei Luo) 


\begin{abstract}
Grain boundaries (GBs) and the spontaneous oxidization around GB regions are inherent features of graphene. In this paper, the thermal conductivity $(\kappa)$ of oxidized polycrystalline graphene $(\mathrm{PG})$ is studied using molecular dynamics simulation. The $\kappa$ of oxidized PG decreases as oxygen $(\mathrm{O})$ coverage increases, which is due to the phonon-defect scattering. However, the relative $\kappa$ reduction of oxidized $\mathrm{PG}$ is much smaller compared to that of oxidized single-crystalline graphene (SG) with the same O coverage when oxidation is localized at the GBs. This is because the GBs themselves are already strong phonon scatterers, such that $\mathrm{O}$ atoms residing at the GBs have far less impact on $\kappa$ as compared to the $\mathrm{O}$ atoms in uniformly oxidized SGs. An effective medium approximation model is developed, which predicts that the influence of both GBs and O atoms on the $\kappa$ of oxidized PG becomes smaller as the grain size increases, such that the $\kappa$ of oxidized PG approaches the $\kappa$ of pristine SG at the large grain limit. The results in this work offer important insights to the thermal transport physics in oxidized PG and provide useful information for the design of graphene-based devices for nanoelectronics and thermal management applications.
\end{abstract}




\section{Introduction}

Graphene, a single atomic carbon (C) layer arranged in a two-dimensional honeycomb lattice, ${ }^{1,}$

${ }^{2}$ has attracted tremendous interest due to its exceptional mechanical, electronic, thermal and optical properties, and its potential for a wide variety of applications. ${ }^{2-5}$ In particular, graphene has a very high thermal conductivity $(\kappa)$, and for suspended single-layer graphene, the measured $\kappa$ is in the range of $1500-5800 \mathrm{~W} / \mathrm{mK}^{5-8}$ Taking advantage of the ultrahigh $\kappa$ of graphene could provide potential solutions to many technological barriers, such as the increasingly severe heat dissipation problem in microelectronics. ${ }^{9,} 10$

Currently, large-scale graphene can be synthesized through chemical vapor deposition (CVD), ${ }^{11-14}$ and the size of CVD graphene can reach up to meters. ${ }^{15}$ However, grain boundaries (GBs) are hard to avoid in CVD graphene, since each grain of the metallic substrate can serve as a nucleation site for individual grains of graphene during the CVD synthesis process. ${ }^{11}$ The structure of GBs in polycrystalline graphene (PG) has been extensively studied both theoretically ${ }^{16-19}$ and experimentally. ${ }^{20,21}$ GBs usually consist of repeating pentagon-heptagon C ring pairs that are separated by regular hexagonal $\mathrm{C}$ rings. The mechanical and thermal transport properties of PG have been well studied as well. ${ }^{19,20,22-25}$ It is reported that even though GBs can greatly weaken the mechanical strength of graphene, ${ }^{19,20}$ they do not deteriorate $\kappa$ significantly. ${ }^{23 \text {, }}$ ${ }^{24}$ One study showed that the interfacial thermal conductance of tilt graphene GBs is in the range of $15-45 \mathrm{GW} / \mathrm{m}^{2} \mathrm{~K}$, which is much higher than that of interfaces made of dissimilar materials, and the $\kappa$ of grains with different sizes and orientations ranges from $700-1500 \mathrm{~W} / \mathrm{mK}^{23}$ Moreover, PG usually has large grain domains, which range from $10 \mu \mathrm{m}$ to $100 \mu \mathrm{m} .{ }^{26}$ Since phonons with mean free paths (MFP) smaller than $10 \mu \mathrm{m}$ already contribute to a very high $\kappa$ $(\sim 3000 \mathrm{~W} / \mathrm{mK}),{ }^{27}$ large-scale $\mathrm{PG}$ is expected to have very high $\kappa$. 
Oxidization can decrease the $\kappa$ of single-crystalline graphene (SG) significantly. ${ }^{28-30}$ A recent study by our group showed that oxygen $(\mathrm{O})$ adatoms, which covalently bind to $\mathrm{C}$ atoms in graphene, can dramatically deform the local structure of SG, leading to very different vibrational characteristics and thus significant phonon-defect scattering. ${ }^{28}$ However, the influence of oxidization on the $\kappa$ of oxidized PG has not been studied. It is reported that the $\mathrm{C}$ atoms at GBs are more chemically reactive than those inside the grains, and thus GBs provide more energetically preferable sites for $\mathrm{O}$ atoms to bind. ${ }^{31}$ This has enabled the observation of graphene GBs using optical microscopes since heating under normal conditions leads to the oxidation of the GBs. ${ }^{31}$ As the GBs themselves can scatter phonons to some extent, the impact of oxidation on the $\kappa$ of PG may be different compared to that in SG.

In this paper, the thermal transport physics of oxidized PG is thoroughly studied using largescale molecular dynamics (MD) simulation. The $\kappa$ of oxidized PG decreases with the O coverage, and the relative $\kappa$ reduction of oxidized PG is much smaller compared to that of the oxidized SG with the same $\mathrm{O}$ coverage when oxidation happens at the GBs. An effective medium approximation (EMA) model is developed to predict the $\kappa$ of oxidized PG with large grain size, and the model shows that the influence of GBs and $\mathrm{O}$ atoms residing at GBs on the $\kappa$ of oxidized PG both become smaller as the grain size increases, such that the $\kappa$ of oxidized PG approaches that of pristine SG at the large grain limit. The results from this study can provide important guidance on the design of graphene-based devices for nanoelectronics and thermal management, especially because it is likely that PG will be used in many practical applications and it will be naturally oxidized when exposed to air.

\section{Results and Discussions}


The structures of pristine SG and oxidized SG are shown in Figure 1(a) and 1(b) respectively. The cyan spheres represent $\mathrm{C}$ atoms and red spheres represent $\mathrm{O}$ atoms. The pristine $\mathrm{SG}$ lattice structure only consists of regular C hexagons without any structural defects. For the oxidized SG, the $\mathrm{O}$ atoms reside at random bridge sites of hexagons on both sides of the SG plane, as it has been reported that the bridge site is the most stable adsorption site for $\mathrm{O}$ atom in graphene. ${ }^{32}$ The structure of PG with a single tilted GB is shown in Figure 1(c). In general, the GB in PG contains pentagon-heptagon ring pairs and slightly distorted hexagonal rings. ${ }^{17,} 20,23,24$ The pentagonheptagon ring pairs are structural defects at GB, and the defect density of GB can be defined as the number of pentagon-heptagon ring pairs over the number of hexagonal rings at $\mathrm{GB} .^{23} \mathrm{In}$ Figure 1(c), the tilted GB only consists of pentagon-heptagon ring pairs, and this GB structure has the highest defect density. ${ }^{23}$ A GB's defect density can also be characterized by the structural mismatch angle $\alpha$ as shown in Figure 1(c). The graphene grains at the left and right sides of GB are tilted in the opposite directions to form this structural mismatch angle. ${ }^{24}$ GBs with larger mismatch angles have higher defect densities. ${ }^{17}$ For the case shown in Figure 1(c), the mismatch angle $\alpha$ is $21.8^{\circ} .^{17}$ 

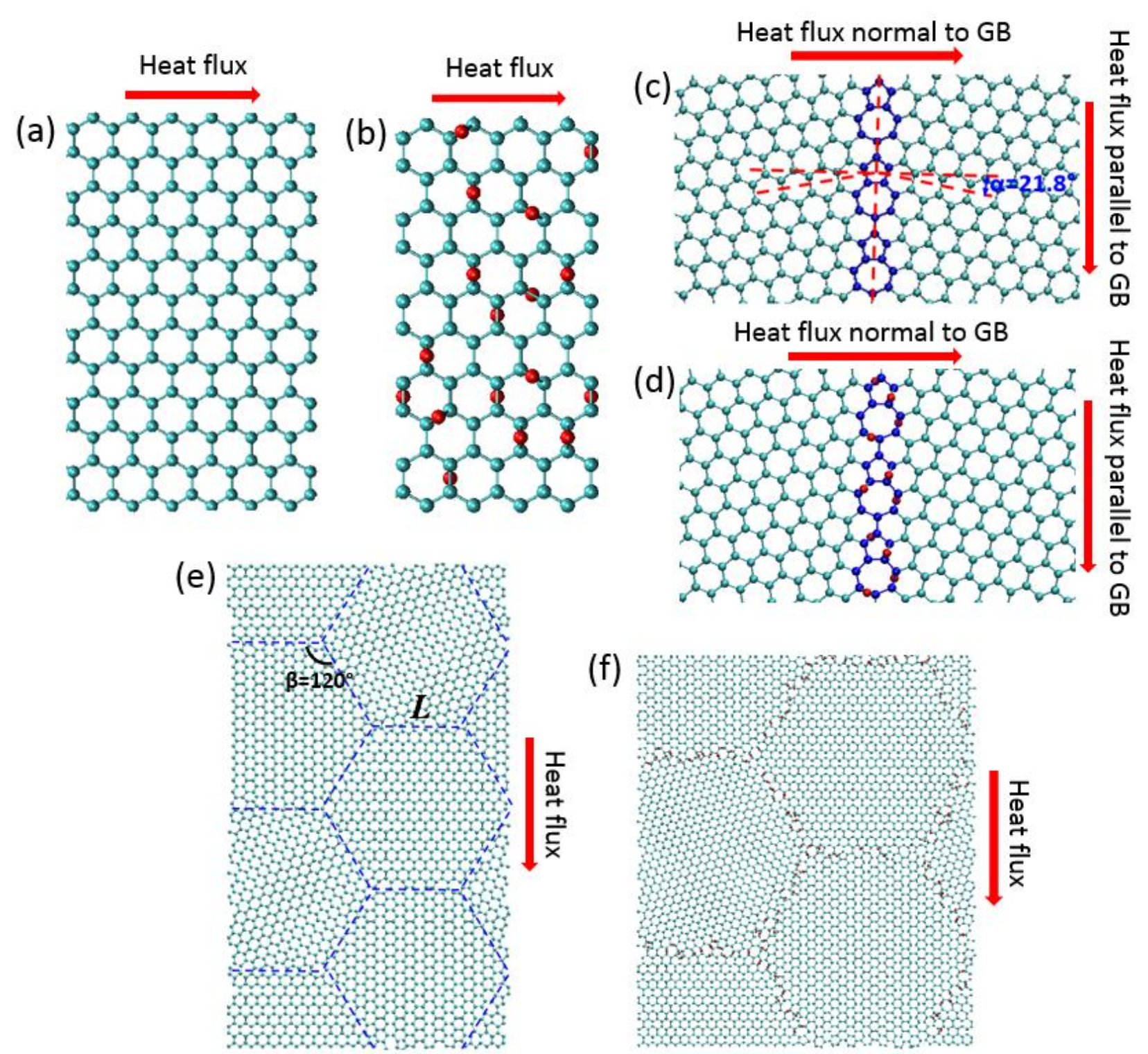

(f)

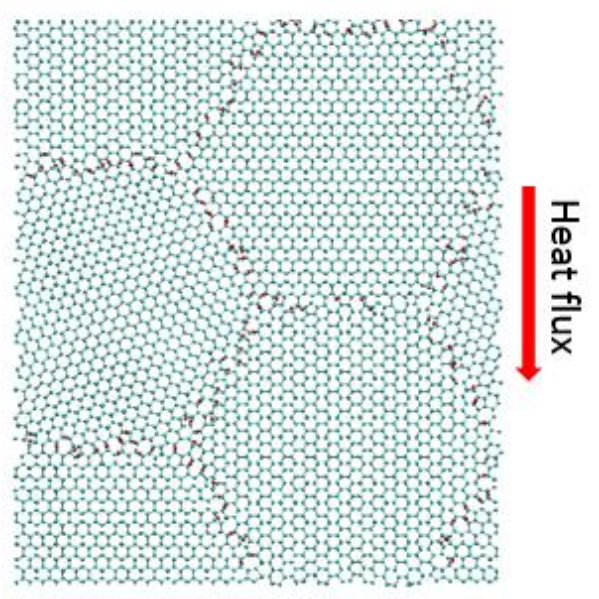

Figure 1. Structures of different graphene samples: (a) Pristine SG; (b) Oxidized SG; (c) Nonoxidized $P G$ with a single tilted $G B$, where the GB has the highest defect density and the corresponding structural mismatch angle $\alpha$ is 21.8 ; (d) Oxidized PG with a single tilted GB and all the $O$ atoms reside at the GB; (e) Non-oxidized $P G$ with regular hexagonal graphene grains with an edge length of L. The blue dashed lines depict the GBs; (f) Oxidized PG with regular hexagonal graphene grains and all the $O$ atoms reside at the GBs. For all the figures, cyan spheres represent $C$ atoms in $S G$ and in graphene grains of non-oxidized and oxidized PG, blue spheres represent the $C$ atoms of $G B$ in non-oxidized and oxidized $P G$, and red spheres represent the $O$ atoms. The red arrows represent the heat flux directions considered in this work. 
The relationship between interfacial thermal conductance of a GB and its defect density has been studied by Bagri et al. and Lu and Guo. ${ }^{23,24}$ Both groups found that the interfacial thermal conductance of GB decreases slightly as the defect density increases. In this work, we only consider a GB with the highest defect density, since it has the largest tunable range for $\mathrm{O}$ coverage of oxidized PG. The $\mathrm{O}$ coverage in this work is defined as the number of $\mathrm{O}$ atoms over the number of $\mathrm{C}$ atoms in graphene. Moreover, since different defect densities of GBs do not cause significant variation of the interfacial thermal conductance, ${ }^{24}$ the knowledge gained in this study should be transferable to other cases with different (smaller) defect densities.

The structure of oxidized PG (with one single tilt GB) is shown in Figure 1(d). All the O atoms reside at the bridge sites of $\mathrm{C}$ rings in the GBs and on both sides of the PG plane. The structures of non-oxidized and oxidized PG with multi-GBs are shown in Figure 1(e) and 1(f). The non-oxidized PG shown in Figure 1(e) consists of regular hexagonal SG grains and GBs with the highest defect density (structural mismatch angle $\alpha$ of $21.8^{\circ}$ ). In preparing the nonoxidized PG structure, successive procedures to optimize the GBs are carried out by adding and removing carbon atoms in the GB regions to adjust the local bonding topologies. The detailed preparation process of non-oxidized PG can be found in Ref [22]. The blue dashed line in Figure 1(e) highlights the position of GBs for clarity, and we define the grain size $L$ as the length of a side of the regular hexagonal SG grains. In the oxidized PG structure shown in Figure 1(f), the O atoms also only reside at the GB regions.

Non-equilibrium MD (NEMD) $)^{28,33,34}$ is used to calculate the $\kappa$ of different graphene structures as well as the interfacial thermal conductance of GBs. In NEMD, the temperatures of two ends of the graphene sample are fixed at different values, and heat flows from the higher temperature end $(315 \mathrm{~K})$ to the lower temperature end $(285 \mathrm{~K})$. The calculation details of NEMD method are 
included in the Method section. For pristine SG and oxidized SG, only one heat flux direction (shown by the red arrows in Figures 1(a) and 1(b)) is considered in NEMD. We ignore the edge chirality (zigzag and armchair edges) effect here, because it does not influence the understanding of the oxidization effect on $\kappa$ as long as the same edge chirality is used for all the non-oxidized and oxidized SG cases. For the PG with one single tilt GB, two heat flux directions (i.e., normal and parallel to the GB) are considered since the thermal transport should be different along these two directions (Figures 1(c) and 1(d)). As for the PG with multiple-GBs (e.g., Figures 1(e) and 1(f)), only one heat flux direction is considered, which should be sufficient to reveal the thermal transport physics.

The calculated $\kappa$ values for different graphene structures are shown in Figure 2(a). These values are obtained from graphene samples with the dimension of $10 \mathrm{~nm} \times 10 \mathrm{~nm}$. The sample size, which is much smaller than the MFPs of some long wavelength acoustic phonons in graphene, imposes a classical size effect on the thermal transport in graphene. ${ }^{35,36}$ This should be the primary reason for the fact that the $\kappa$ values shown in Figure 2(a) are much smaller than the experimental values. ${ }^{5-8}$ Another reason could be the use of Reactive Empirical Bond Order (REBO) potential ${ }^{37-39}$ in the NEMD simulation, which is known to under-predict the $\kappa$ of graphene even when extrapolating graphene's length to infinite. ${ }^{28}$ 
(a)

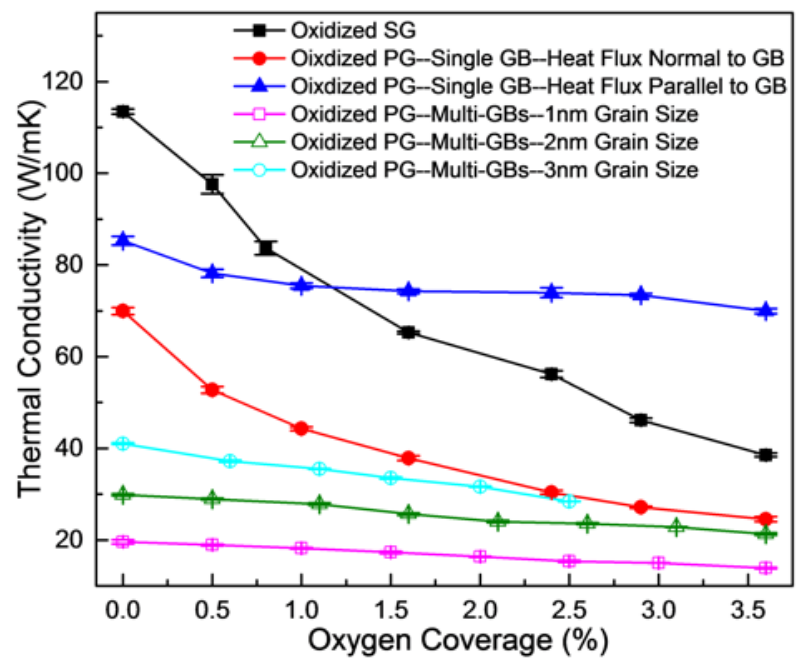

(b)

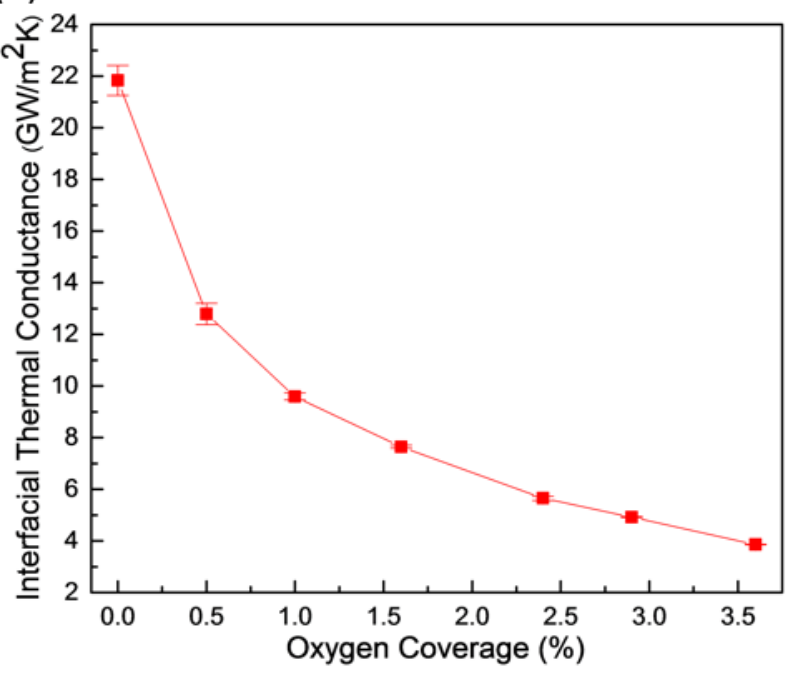

Figure 2. (a) $\kappa$ of oxidized $S G$ and $P G$ with different grains and $G B$ dimensions as a function of the $O$ coverage. For the oxidized $P G$, all the $O$ atoms reside at the GBs. All the cases studied here have the same dimension of $10 \mathrm{~nm} \times 10 \mathrm{~nm}$. (b) The interfacial thermal conductance of oxidized $G B$ as a function of $O$ coverage.

The $\kappa$ of oxidized SG as a function of $\mathrm{O}$ coverage is shown by the solid black squares in Figure 2(a), where the zero O coverage case corresponds to pristine SG. It is clearly shown that the $\kappa$ of oxidized SG decreases rapidly with the O coverage. When the $\mathrm{O}$ coverage is $3 \%$, the $\kappa$ is only $39.1 \%$ of that of pristine SG. The reason for this sharp decrease of $\kappa$ is the strong phonondefect scattering caused by the adsorbed $\mathrm{O}$ atoms, which has been explained in detail in Ref. [28].

The $\kappa$ of oxidized $\mathrm{PG}$ with one single tilt $\mathrm{GB}$ as a function of $\mathrm{O}$ coverage is shown by the solid red circles and blue triangles in Figure 2(a). The red circles represent the cases with heat flux normal to the GB, and the blue triangles represent the cases with heat flux parallel to the GB (Figure 1(d)). We denote the $\kappa$ in these two directions as $\kappa_{\perp}$ and $\kappa_{\|}$respectively. First, we observe that when the $\mathrm{O}$ coverage is zero, corresponding to non-oxidized PG, $\kappa_{\|}$and $\kappa_{\perp}$ are both smaller 
than that of pristine SG. This is due to the fact that the structural defects offered by the GB itself are effective phonon scatterers. Moreover, for non-oxidized PG, $\kappa_{\perp}$ is smaller than $\kappa_{\|}$. When the heat flux is normal to the GB, more phonons that transmit across the sample scatter with the GB than the case in which the heat flux is parallel to GB, thus resulting in a smaller $\kappa$. Secondly, both $\kappa_{\perp}$ and $\kappa_{\|}$decrease with $\mathrm{O}$ coverage, and the oxidation has much larger impact on $\kappa_{\perp}$ than on $\kappa_{\|}$. Compared to the $\kappa$ of oxidized SG (black squares), $\kappa_{\|}$has a much slower decreasing trend with O coverage, while $\kappa_{\perp}$ decreases at a similar rate as oxidized SG. When the O coverage is $3 \%, \kappa_{\|}$ decreases by $14.7 \%$ compared to its pristine case, and $\kappa_{\perp}$ decreases by $62.6 \%$. It is noted that we only consider one specific sample dimension $(10 \mathrm{~nm} \times 10 \mathrm{~nm})$ here. When we increase the sample size, we do not expect different trend of $\kappa_{\perp}$ as a function of $\mathrm{O}$ coverage, since the GB and oxidation effect is strong. However, the variation trend of $\kappa_{\|}$would tend to be more flat when we increase the sample size. This is because, when the heat flux direction is parallel to GB, the GB and oxidation effect will not be strong enough to overcome the size effect, and the larger sample will be influenced less by the oxidation effect.

It was somewhat unexpected that the $\mathrm{O}$ atoms absorbed at the GB can have such significant additional impact on $\kappa_{\perp}$ even though the GB itself already scatters phonons. The implication is that the behavior of $\mathrm{O}$ atoms as defects is greater than (or at least comparable to) the structural defects in the GB due to $\mathrm{C}$ reorganization alone. To further investigate the effect of oxidation on $\kappa_{\perp}$, we calculate the interfacial thermal conductance of the GB for oxidized PG at different $\mathrm{O}$ coverages. The calculation details are included in the Method section, and the results are shown in Figure 2(b), where it is clear that the interfacial thermal conductance is also strongly dependent on the $\mathrm{O}$ coverage, explaining the sharp decrease in $\kappa_{\perp}$ for oxidized PG (Figure 2(a), solid red circles). 
To further explore the physical origin of the effect of GB and oxidation on the thermal transport, the vibrational features of different atoms around the GB, which are characterized by their vibrational power spectra (VPS), are analyzed, as shown in Figure 3. Figure 3(a) shows the positions of $\mathrm{C}$ and $\mathrm{O}$ atoms studied in the VPS analyses, and the color code for each of the different atoms in Figure 3(a) is the same as their corresponding VPS shown in Figures 3(b)-(l). The VPS is calculated by taking Fourier transform of the autocorrelation function of atom's velocity, and the calculation details are described in the Method section. Figure 3(b)-(f) show the VPS of C atoms in the GB or neighboring to the GB in non-oxidized PG. Figure 3(g) shows the VPS of O atoms, and Figure 3(h)-(l) show the VPS of $\mathrm{C}$ atoms in the GB or neighboring to the GB in oxidized PG with a O coverage of 3.5\%. In each figure, the VPS of $\mathrm{C}$ atoms in pristine SG (black curves) is plotted as the reference to highlight the changes in VPS due to the GB and oxidation. 
(a)

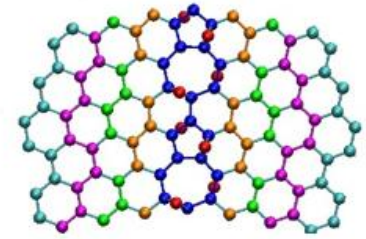

Non-oxidized PG

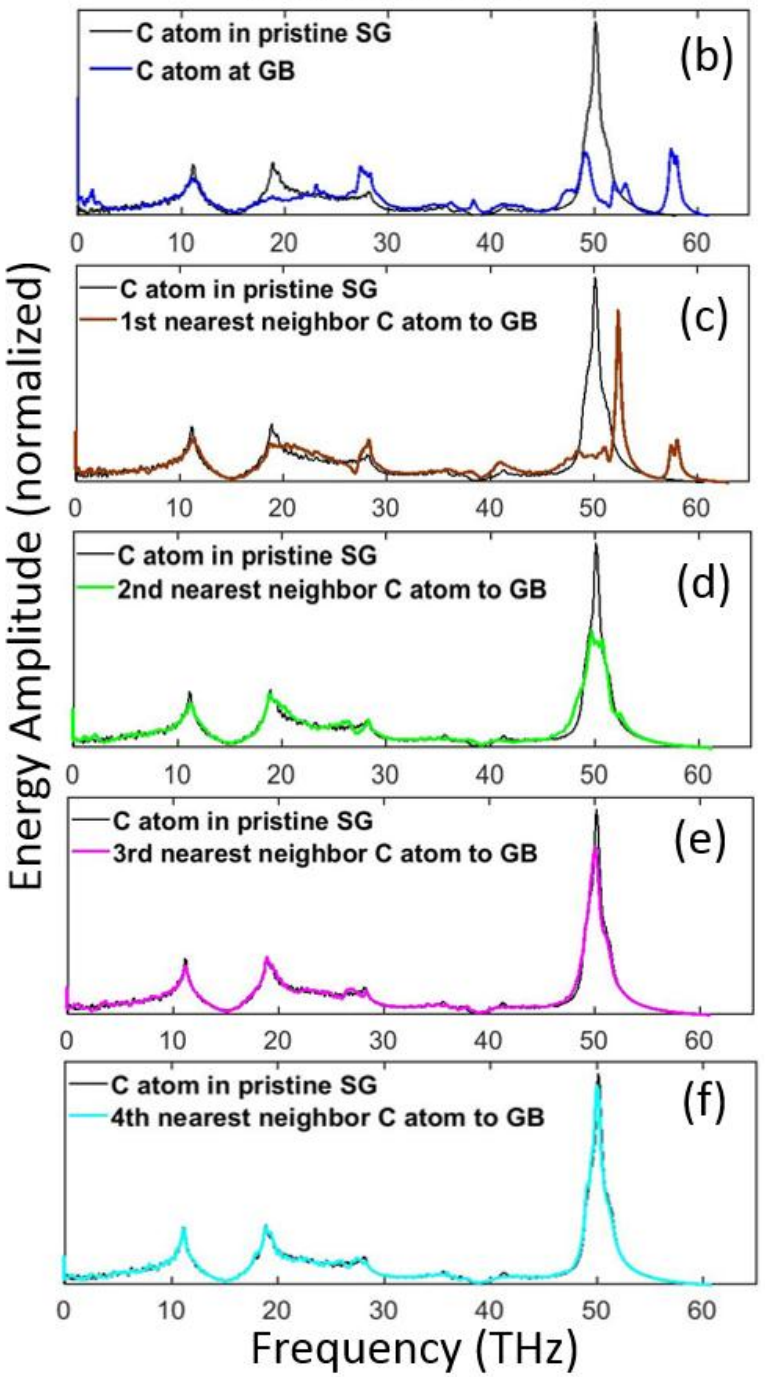

Oxidized PG-3.5\% O atoms

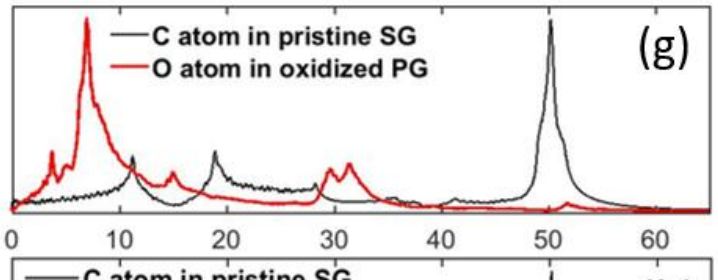

(h)
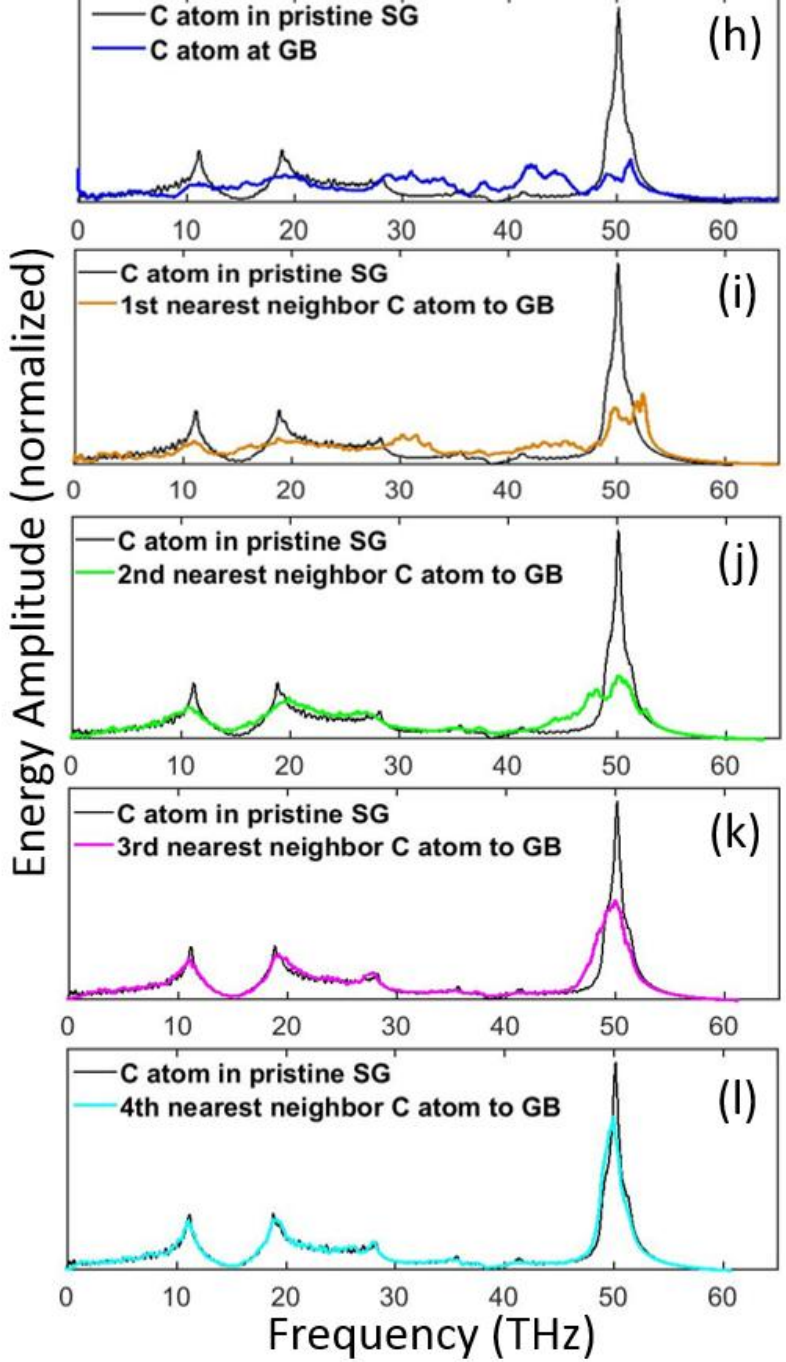

Figure 3. (a) The illustrated structure of oxidized $P G$, which indicates the positions of $C$ and $O$ atoms studied in the VPS analyses. The red spheres represent the $O$ atoms, the blue spheres represent the $C$ atoms at $G B$, and the orange, green, magenta and cyan spheres represent the $C$ atoms that are the first, second, third and fourth nearest neighbors to GB respectively. (b)-(f) $V P S$ of $C$ atoms at GB and $C$ atoms that are the first, second, third and fourth nearest neighbors to GB in non-oxidized PG. ( $g$ ) VPS of $O$ atoms. (h)-(l) VPS of $C$ atoms at GB and $C$ atoms that are the first, second, third and fourth nearest neighbors to $G B$ in oxidized $P G$ with an $O$ 
coverage of $3.5 \%$. The color code of the VPS in panels $(b)-(l)$ is the same as that for the different $C$ and $O$ atoms in panel (a). The VPS of C atoms in pristine SG (black curve) is plotted in panels (b)-(l) for comparison.

We first discuss the VPS of $\mathrm{C}$ atoms in non-oxidized PG. It can be seen from Figure 3(b) that the VPS of C atoms in GB (blue curve) is very different from that of pristine SG (black curve) due to their different bonding environments. In the GB, C atoms are in pentagon-heptagon ring pairs (see Figure 1(c)), while in pristine SG, C atoms are in regular hexagonal rings (see Figure 1(a)). Figure 3(c) and (d) show that the VPS of C atoms which are the first and second nearest neighbors to GB are also different from the black curve, meaning that the GB can also change the atomic vibrational characteristics of $\mathrm{C}$ atoms around $\mathrm{GB}$, despite the fact that these $\mathrm{C}$ atoms have similar bonding environment as the $\mathrm{C}$ atoms in pristine $\mathrm{SG}$ (i.e., in hexagonal rings). We attribute this to local structural deformation around the GB, which changes the vibrational characteristics of the surrounding C atoms. Figures 3(e) and (f) show the VPS of the third and fourth nearest neighboring $\mathrm{C}$ atoms to $\mathrm{GB}$, which appear to recover the behavior of $\mathrm{C}$ atoms in pristine graphene (the black curve). Therefore, we conclude that the structural defect at the GB impacts the vibrational characteristics of $\mathrm{C}$ atoms up as far as its second nearest neighbors.

The VPS of $\mathrm{O}$ atoms in oxidized PG is shown in Figure 3(g), and there is significant difference between the red and black curves. It is not surprising that the $\mathrm{O}$ atom has a very different VPS from the $\mathrm{C}$ atoms in pristine SG as it has both a significantly different mass and bond strength. Perhaps more importantly is that the strong covalent bonds that $\mathrm{O}$ atoms form with $\mathrm{C}$ atoms also change the vibrational characteristics of the $\mathrm{C}$ atoms. In our prior work on oxidized SG, we showed this impact can extend as far as the third nearest neighbor of the C-O bonding site. ${ }^{28}$ Here we show that the VPS of a C atom in the GB of oxidized PG (see Figure 
3(h)) is different from both the VPS of C atoms in pristine SG and the VPS of C atoms in the GB of non-oxidized PG (see Figure 3(b)). Two mid-frequency vibrational peaks (at 11 and $19 \mathrm{THz}$ ) of $\mathrm{C}$ atoms are significantly suppressed by the oxidation. This will lead to a mismatch in the VPS between the $\mathrm{C}$ atoms in the $\mathrm{GB}$ and those inside the grain, and thus could be one reason for the decrease in $\kappa_{\perp}$ caused by oxidization (shown by the red circles in Figure 2(a)).

From Figures 3(i) to (j), we observe that the VPS of the first and second nearest neighbor C atoms of $\mathrm{GB}$ in oxidized $\mathrm{PG}$ are also different from that of $\mathrm{C}$ atoms in pristine $\mathrm{SG}$. It is interesting to note that, due to the existence of $\mathrm{O}$ atoms, the VPS of the third nearest neighbor C atoms of GB in oxidized PG is also different from that of $\mathrm{C}$ atoms in pristine SG (see Figure $3(\mathrm{k})$ ), illustrating that the adsorption of $\mathrm{O}$ atoms can broaden the influence range of GB. For this oxidized PG (O coverage of 3.5\%), the influence range of the oxidized GB is up as far as its third nearest neighbor $\mathrm{C}$ atoms. Beyond this range, the VPS of $\mathrm{C}$ atoms is almost the same as that of the pristine SG (Figure 3(1)). We note that this is consistent with our prior work, ${ }^{28}$ and explains why $\kappa_{\perp}$ decreases so significantly with $\mathrm{O}$ coverage as the impact of oxygen on the phonon behavior is greater than the structural defects in GB caused by the $\mathrm{C}$ reorganization.

The $\kappa$ values of oxidized PG with regular hexagonal grains are shown as the hollow cyan circles (grain size of $1 \mathrm{~nm}$ ), hollow green triangles (grain size of $2 \mathrm{~nm}$ ), and hollow magenta squares (grain size of $3 \mathrm{~nm}$ ) in Figure 2(a). It is worth emphasizing again that all the simulation results shown in Figure 2(a) are for simulation domains with the same dimension of $10 \mathrm{~nm} \times 10$ $\mathrm{nm}$, so that the same size effect applies to all cases, enabling fair comparison between cases. We can see that for all oxidized PGs with regular hexagonal grains, the $\kappa$ values are smaller than those of the oxidized SG and oxidized PG with a single tilted GB. This is because, with the same domain size, the PG samples with hexagonal grains have more GBs, and there are more phonon- 
GB and phonon-O atom scatterings. Due to the same reason, PGs with smaller grain sizes and thus higher GB densities have smaller $\kappa$, as the $1 \mathrm{~nm}$ grain case has the lowest $\kappa$. Moreover, for all these three cases, $\kappa$ decreases as $\mathrm{O}$ coverage increases. However, they decrease much slower than that of oxidized SG and oxidized PG with a single tilted GB $\left(\kappa_{\perp}\right)$, since parts of the GBs are not orientated perpendicular to the heat flux direction.

So far, we have only investigated the $\kappa$ of non-oxidized and oxidized PG with relatively small grain sizes (grain sizes of 1, 2 and $3 \mathrm{~nm}$ ) and small domains (10 $\mathrm{nm} \times 10 \mathrm{~nm})$. We note, however, that typically realistic grain sizes of PG are usually at the micrometer scale. ${ }^{15,26}$ However, it would be too computationally expensive to use MD simulation to calculate the $\kappa$ of PG with grain sizes at the micrometer scale. Thus, to extend our MD simulation results to larger grain sizes, we develop here an effective medium approximation (EMA) model that takes the MD simulation results of small grain and domain sizes as inputs to predict the $\kappa$ of non-oxidized and oxidized PG with any arbitrary grain size.

Generally speaking, EMA model is an approximation method to calculate the macroscopic properties of composite materials by averaging the properties of the constituents that make up the composite. ${ }^{40-42}$ In our EMA model, PG (both non-oxidized and oxidized) is considered as a composite materials that consists of two phases - graphene grains and GBs (or oxidized GBs). We combine the anisotropic (serial and parallel) models of $\kappa$ with weighting factors to calculate the $\kappa$ of non-oxidized and oxidized PG. ${ }^{41,42}$ The $\kappa$ values of graphene grains and GBs are obtained from MD simulations and used as inputs for the EMA model. The detailed description of the EMA model is included in the Method section.

Firstly, to validate our EMA model, we calculate the $\kappa$ of non-oxidized and oxidized PG with grain size of 1, 2, 3 and $5 \mathrm{~nm}$ using the EMA model, and compare the results with those 
calculated by MD simulations (see Table 1 and 2). We can see that the $\kappa$ values calculated by the EMA model are very close to those calculated by MD simulations (the largest difference between these two methods is below $\sim 7.7 \%$ ). Therefore we believe that the EMA model can give good prediction of the $\kappa$ of non-oxidized and oxidized PG.

Table 1. The $\kappa$ of non-oxidized PG with grain size of 1,2,3 and 5nm calculated by EMA model and MD simulations.

\begin{tabular}{|c|c|c|c|c|}
\hline Grain Size (nm) & 1 & 2 & 3 & 5 \\
\hline$\kappa$-- EMA model & $15.6 \pm 0.4$ & $34.4 \pm 0.9$ & $53.9 \pm 1.1$ & $80.6 \pm 1.2$ \\
$(\mathrm{~W} / \mathrm{mK})$ & & & & \\
\hline$\kappa--$ MD simulation & $14.5 \pm 0.3$ & $32.9 \pm 0.4$ & $50.4 \pm 0.2$ & $83.4 \pm 0.3$ \\
$(\mathrm{~W} / \mathrm{mK})$ & & & & \\
& & & & \\
\hline
\end{tabular}

Table 2. The $\kappa$ of oxidized PG with grain size of 1, 2 and 3nm calculated by EMA model and MD simulations.

\begin{tabular}{|c|c|c|c|}
\hline Grain Size (nm) & 1 & 2 & 3 \\
\hline$\kappa--$ EMA model & $4.8 \pm 0.04$ & $16.7 \pm 0.5$ & $30.9 \pm 0.7$ \\
$(\mathrm{~W} / \mathrm{mK})$ & & & $31.8 \pm 0.4$ \\
\hline$\kappa-$ MD simulation & $5.2 \pm 0.1$ & $17.6 \pm 0.1$ & \\
\hline
\end{tabular}


The $\kappa$ of non-oxidized and oxidized PG with hexagonal grains calculated by the EMA model as a function of the grain size are shown in Figure 4(a). The black squares represent the $\kappa$ of nonoxidized PG, and the red circles represent $\kappa$ of oxidized PG with all $\mathrm{O}$ atoms residing at the GBs with a full coverage. A full $\mathrm{O}$ coverage means that all the potential adsorption sites of the GBs are saturated by $\mathrm{O}$ atoms. We can see that for both the non-oxidized and oxidized PG, $\kappa$ increases quickly with the grain size. This is because the larger grain size can accommodate longer phonon MFPs, and thus the grains have larger thermal conductivity ( $\kappa_{1}$ in EMA model). In our previous work, we showed that for pristine SG, $\kappa$ converges to a value of $925.6 \mathrm{~W} / \mathrm{mK}$ at large size, ${ }^{28}$ as calculated by MD simulation with the REBO potential. We show this value here as the black dashed line, and it is clear that when the grain size is on the micrometer scale, the $\kappa$ of PG and oxidized PG both approach that of the pristine SG (black dashed line). At these large grain sizes, the GBs are spaced further than the longest phonon MFP, and thus GB and $\mathrm{O}$ atoms (residing at GBs) scatterings have negligible influence on the overall thermal transport.
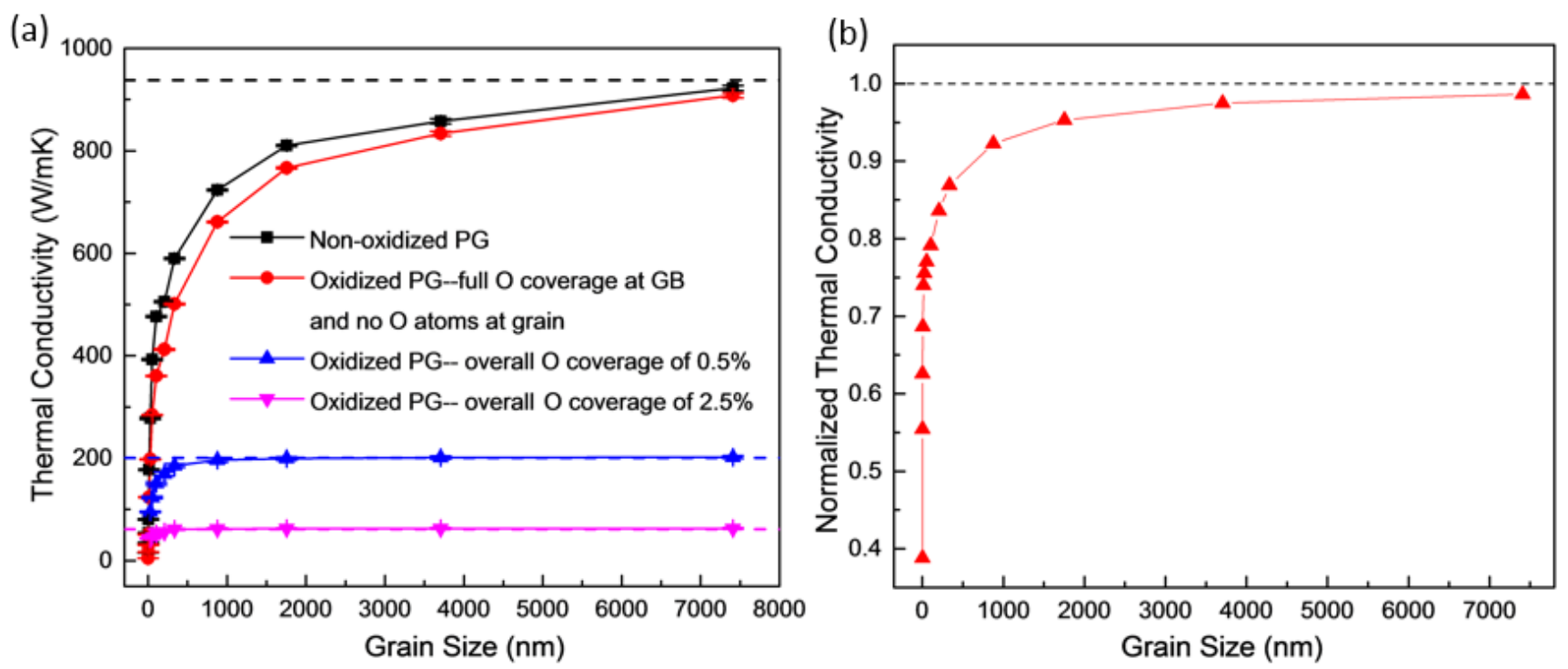
Figure 4. (a) $\kappa$ of non-oxidized and oxidized $P G$ with hexagonal grains as a function of grain size calculated by the EMA model. The black squares represent the $\kappa$ of non-oxidized PG. The red circles represent the $\kappa$ of oxidized $P G$ with all $O$ atoms residing at the $G B$ with a full coverage. The blue upper triangles and magenta lower triangles represent $\kappa$ of oxidized $P G$ with $O$ coverage of $0.5 \%$ and $2.5 \%$ respectively. For these two cases, the $O$ atoms reside at both GBs and inside the grains. The black, blue and magenta dashed lines indicate the converged $\kappa$ of pristine $S G$, oxidized $S G$ with $O$ coverages of $0.5 \%$ and $2.5 \%$ with infinite sizes calculated by the MD simulations. ${ }^{28}$ (b) Normalized $\kappa$ of oxidized $P G$ (red circles in panel (a)) against that of nonoxidized PG (black squares in panel (a)) as a function of the grain size.

Importantly, as the grain size increases, the number of $\mathrm{C}$ atoms increases faster than that of $\mathrm{O}$ atoms. Therefore the ratio of $\mathrm{O}$ atoms to the total number of $\mathrm{C}$ atoms decreases with the grain size, and it approaches zero when the grain size goes to infinity. For this reason, the two curves (black squares and red circles) asymptotically approach each other with their difference approaching zero. We show this more clearly in Figure 4(b) where we plot the ratio of the two curves, the normalized $\kappa$ of oxidized PG against that of non-oxidized PG, as a function of the grain size, and the ratio asymptotically approaches 1 . However, the impact of oxidation of GB is significant when the grain size is small $(<100 \mathrm{~nm})$. In reality, the grain sizes of PG are usually on the micrometer scale. Our calculation shows that, for such large grain sizes, the influence of oxidation on the thermal transport can be very small if all the $\mathrm{O}$ atoms reside at the GB. Therefore under most realistic conditions we do not expect large $\kappa$ reduction for the oxidized PG, considering the fact that $\mathrm{C}$ atoms at GBs are more chemically reactive than those inside the grains, and thus GBs provide more energetically preferable sites for $\mathrm{O}$ atoms to bind. ${ }^{31}$

In the cases that PG is excessively oxidized beyond the situation where $\mathrm{O}$ atoms only react with GBs, $\mathrm{O}$ atoms can bond to $\mathrm{C}$ atoms inside the grains. The blue upper triangles and magenta 
lower triangles in Figure 4(a) show the $\kappa$ of oxidized PG with $\mathrm{O}$ atoms residing at both GBs and within the graphene grains. Here, we have assumed that $\mathrm{O}$ atoms will first occupy all possible bonding sites in the GBs before they start to bind to the $\mathrm{C}$ atoms inside the grains. Such an assumption is based on the aforementioned fact that $\mathrm{C}$ atoms in the $\mathrm{GB}$ are much more reactive than those in the grain boundaries. ${ }^{31}$ Under such an assumption, $\kappa_{1}$, the thermal conductivity of grain, decreases as the $\mathrm{O}$ coverage increases according to our previous calculations in Ref. [28], but $\kappa_{2}$, the effective thermal conductivity of the GB, is held constant since we have assumed that the GB is saturated with oxidation.

The blue upper triangles represent the cases with an overall $\mathrm{O}$ coverage of $0.5 \%$, and the magenta lower triangles represent the cases with an overall $\mathrm{O}$ coverage of $2.5 \%$. We can see that the $\kappa$ of these two cases both increase with grain size at first, and then become saturated quickly. The saturated $\kappa$ of these two cases are 202.9 and $62.9 \mathrm{~W} / \mathrm{m}^{2} \mathrm{~K}$ respectively, which are much smaller than the converged $\kappa$ of pristine SG. Such a large difference originates from the much lower $\kappa$ of the PG grains when oxidized. However, both $\kappa$ values approach the respective values of oxidized SG (shown as the blue and magenta dashed lines) with the same O coverages, ${ }^{28}$ and the convergences happen at much smaller grain sizes compared to the cases where $\mathrm{O}$ atoms only reside at GB. This is because the grain size effect due to boundary scattering is less pronounced when the grains are oxidized, where the phonon-oxygen defect scattering has already effectively reduced the MFP of phonons.

\section{Conclusions}

The $\kappa$ of non-oxidized and oxidized PG with different GB geometries and dimensions are studied using MD simulation and an EMA model in this work. It is revealed that the $\kappa$ of 
oxidized PG decreases as the O coverage increases, which is due to the phonon-defect (GB and $\mathrm{O}$ adatoms) scattering. However, the relative $\kappa$ reduction of oxidized PG is much smaller compared to that of the oxidized SG with the same O coverage. Since the O atoms of oxidized PG mostly reside at the GBs, which already scatter phonons, the impact of oxidation on $\kappa$ is less significant compared to that in SG. The vibrational characteristics of atoms around the GB in non-oxidized and oxidized PG are studied by the VPS analyses, which confirms that the defectinduced vibration spectra mismatch causes the reduction of $\kappa$. An effective EMA model is developed based on the serial and parallel models. Taking the MD simulation results as inputs, this EMA model predicts that the influence of the GB and $\mathrm{O}$ atoms on the $\kappa$ of oxidized PG becomes smaller as the grain size increases, and the $\kappa$ of oxidized PG approaches that of the pristine $\mathrm{SG}$ at the large grain limit if all the $\mathrm{O}$ atoms reside at the GBs. However, when the grains themselves are oxidized, the $\kappa$ of oxidized PG is further reduced. This work thoroughly studies the influence of oxidization on the $\kappa$ of $\mathrm{PG}$, and the results from this work can provide useful information for the design of graphene-based devices for nanoelectronics and thermal management applications. 


\section{Method}

\subsection{Molecular Dynamics Simulations}

In this work, the large-scale atomic/molecular massively parallel simulator (LAMMPS) ${ }^{43}$ is used to perform all the MD simulations. The REBO potential parameterized for the C-O systems (REBO-CO potential), ${ }^{37}$ which is an expansion of the original REBO potential, ${ }^{38,}{ }^{39}$ is used to model the interatomic interactions. The REBO-CO potential has been used to predict the thermal and structural behaviors of graphene oxide and oxidized carbon nanotube with reasonable accuracy. ${ }^{28,44}$

To obtain the optimized structure of each graphene structure (SG and PG), the total energies are first minimized using the steepest descent algorithm, ${ }^{45}$ then followed by a constant number, constant pressure, and constant temperature ensemble (NPT) simulation at $300 \mathrm{~K}$ and 1 atm to further relax the sample structure. The Nose-Hoover method ${ }^{46,47}$ is used for the thermostating/barostatting in the NPT ensemble. During this structural optimization process, periodic boundary conditions are used in the $x$ and $y$ directions to simulate an infinite graphene sample, and the isolated boundary condition is used in the $z$ direction.

Based on the optimized structures, NEMD is performed to calculate the thermal properties of each graphene structure. The isolated boundary condition is used in the $x, y$ and $z$ directions to model a graphene sample with finite size. It is noted that the positions of the end atoms in the $x$ direction are fixed to prevent sample drifting and deformation during the NEMD simulation. A constant number, constant volume, and constant temperature ensemble (NVT) is first performed for $1 \mathrm{~ns}$ to equilibrate the graphene sample, and then a constant number, constant volume, and constant energy ensemble (NVE) is performed to calculate the thermal properties. In the NVE ensemble, a temperature gradient is imposed across the simulation domain by setting the 
temperatures of the two ends in the $x$ direction at different values using Langevin thermostats. ${ }^{48}$ For all the simulations, the temperature of heat source (higher temperature end) and the temperature of heat sink (lower temperature end) are maintained at 315 and $285 \mathrm{~K}$ respectively. The energies added into the heat source and subtracted from the heat sink are recorded to calculate the heat flux flowing across the structure.

To reach steady state, the NVE ensemble usually needs to be performed for at least 2 ns. When steady state is reached, the heat flux $(J)$ is calculated using:

$$
J=(d Q / d t) / S
$$

where $d Q / d t$ is the average of the energy input and output rates of the heat source and heat sink, and $S$ is the cross-sectional area. To calculate the cross-sectional area of graphene, we take $3.35 \AA$, which is the inter-layer distance of graphite, as the thickness of graphene. At the steady state, the overall thermal conductance $(G)$ of graphene sample is calculated using:

$$
G=\frac{J}{\Delta T}
$$

where $\Delta T$ is the overall temperature drop across the graphene sample. Then the overall $\kappa$ is calculated by multiplying $G$ by the effective total length of graphene sample. It is noted that the artificial boundary effect, ${ }^{49,50}$ which is caused by the Langevin thermostats ${ }^{48}$ at sample's two ends, is eliminated when calculating $\kappa$. The detailed method of eliminating the artificial boundary effect can be found in Ref. [49]. To calculate the interfacial thermal conductance of GB (Figure 2(b)), the local temperature drop at GB is used in Equation (2). 
For each NEMD simulation in this study, four $\kappa$ values are obtained from four different time blocks at steady state, and the final value is the average of these four values with the error bar being the standard deviation. A time step of $0.5 \mathrm{fs}$ is used for all the MD simulations.

\subsection{Vibrational Power Spectra}

The VPS is calculated by taking the Fourier transform of the autocorrelation function of atom's velocity:

$$
I(\omega)=\int_{-\infty}^{\infty} \exp (-2 \pi \mathrm{i} \omega t) \mathrm{G}(t) d t
$$

where $\omega$ is the vibrational frequency, $t$ is the correlation time and $\mathrm{G}(t)=\langle v(t) v(0)\rangle$ is the velocity autocorrelation function. For each case in Figure 3 (b) to (1), the VPS of 15 equivalent atoms are calculated and the final VPS is the averaged VPS of these 15 equivalent atoms.

\subsection{Effective Medium Approximation Model}

An EMA model is developed to calculate the effective $\kappa$ of non-oxidized and oxidized PG with arbitrary grain sizes. This model is a combination of the serial and parallel models proposed in Ref. [41, 42], which are used to deal with the cases in which the heat flux is normal and parallel to the GB respectively. For the serial model, the $\kappa_{\perp}$ is expressed as:

$$
\kappa_{\perp}=\frac{1}{(1-f) / \kappa_{1}+f / \kappa_{2}} .
$$

and for the parallel model, the $\kappa_{\|}$is expressed as: 


$$
\kappa_{\square}=(1-f) \kappa_{1}+f \kappa_{2} .
$$

In these two equations, $\kappa_{1}$ and $\kappa_{2}$ are the thermal conductivities of components 1 and 2 in a composite material, and $f$ is the volume fraction of component 2 relative to the entire composite structure.

PG (non-oxidized and oxidized PG) can be approximately regarded as a composite which consists of two phases: graphene grains and GBs (or oxidized GBs). Therefore $\kappa_{1}$ and $\kappa_{2}$ in Equations (4) and (5) are the $\kappa$ of a graphene grain and the $\kappa$ of a GB (or oxidized GB), respectively. To obtain the effective $\kappa$ of PG (or oxidized PG) with regular hexagonal grains, we combine the serial and parallel models (Equation (4) and (5)) by weighting factors $a$ and $b$ as:

$$
\kappa=a \kappa_{\perp}+b \kappa_{\square}
$$

where $a$ and $b$ are based on the specific geometries of the hexagonal grain. For PG (or oxidized PG) with regular hexagonal grains, the inner angle $(\beta)$ of the hexagonal grain is $120^{\circ}$ (see Figure 1(e)) and the effective length of a GB in one grain is $2 L$ and $1.73 L$ for the cases where the GB is normal and parallel to the heat flux respectively. Here $L$ is the edge length of the graphene grain (see Figure 1(e)). Therefore the weighting factors $a$ and $b$ are 0.54 and 0.46 respectively.

To use this EMA model to predict the effective $\kappa$ of non-oxidized and oxidized PG, the values of $\kappa_{1}$ and $\kappa_{2}$ are needed as inputs. The $\kappa$ of pristine SG with a squared-shape as a function of the squared edge length is calculated by NEMD simulations, and the results are shown in Figure 5 (blue triangles). The structure of squared pristine SG is shown in the inset (a) in Figure 5. The $\kappa$ value of squared SG with the same area as the hexagonal graphene grain is used as $\kappa_{1}$ in our EMA model. For example, the hexagonal grain in PG with 1nm edge length has an area of 2.6 
$\mathrm{nm}^{2}$. The $\kappa$ of a squared SG with the area of $2.6 \mathrm{~nm}^{2}$ is calculated, and the value is used as $\kappa_{1}$ in the EMA model to calculate the $\kappa$ of PG (or oxidized PG) with hexagonal grain size of $1 \mathrm{~nm}$.

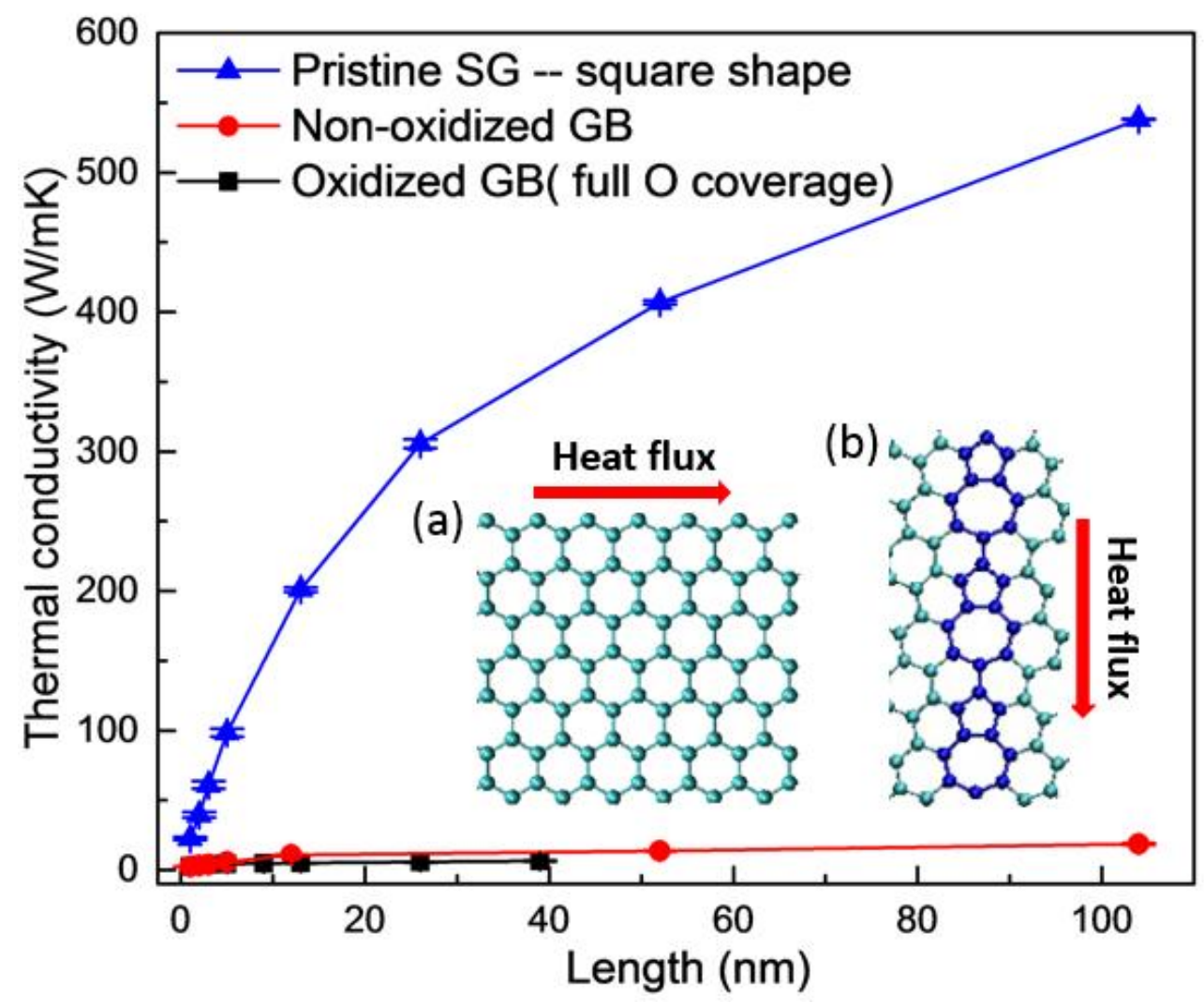

Figure 5. $\kappa$ of pristine $S G$ with squared shape, non-oxidized and oxidized GBs (heat flux is parallel to GB) as a function of their respective characteristic length. The oxidized GB has a full $O$ coverage. The characteristic length of pristine $S G$ is the square edge length, and the characteristic lengths of non-oxidized and oxidized GBs are their total lengths along the heat flux direction. The illustrated structures of pristine $S G$ with squared shape and non-oxidized GB are shown in the insets $(a)$ and $(b)$.

In Figure 5, it can be seen that the $\kappa$ of a squared SG increases with its edge length, and the increasing trend is still not converged when the grain size reaches $100 \mathrm{~nm}$. This is due to the classical size effect of graphene, which has been well discussed in Ref. [28]. For graphene grains 
with sizes larger than the cases shown in Figure 5, we take the $\kappa$ values of pristine SG from Ref [28] as $\kappa_{1}$ in our EMA model.

$\kappa_{2}$ in our EMA model is the nominal $\kappa$ of a GB or a oxidized GB. For the serial model (Equation (4)), $\kappa_{2}$ is the thermal conductivity of the GB (or oxidized GB) when the heat flux is normal to the GB, which is referred as $\left.\kappa_{2}\right\lrcorner \kappa_{2} \perp$ is calculated by multiplying the interfacial thermal conductance of GB (oxidized GB) by its effective width. We approximate the effective widths of a GB and a oxidized GB to be 8.3 and $13.1 \AA$, respectively, according to the VPS results. Our VPS results show that a GB influences the atomic vibrational characteristics up as far as its second (or third for oxidized GBs) nearest neighbor $\mathrm{C}$ atoms. Therefore, we take the average distance between the second (or third) nearest neighbor $\mathrm{C}$ atoms on each side of a GB (or oxidized GB) as the effective length of the GB (or oxidized GB). It is noted that the grain size effect on $\kappa_{2}$ is ignored in our model and the same $\kappa_{2} \perp$ value is used for different grain sizes. We calculate $\kappa_{2 \perp}$ of non-oxidized GB with the grain sizes (edge length) of 5, 10 and $25 \mathrm{~nm}$, and obtain the results of $15.9,16.2$ and $16.8 \mathrm{~W} / \mathrm{m}^{2} \mathrm{~K}$ respectively. The very small difference in $\kappa_{2 \perp}$ for these three cases validates our approximation on the constant $\kappa_{2 \perp}$.

For the parallel model (Equation (5)), $\kappa_{2}$ is the thermal conductivity of GB (oxidized GB) when the heat flux is parallel to it, which is referred as $\kappa_{2 \|} \cdot \kappa_{2} \|$ is calculated by NEMD on isolated GB ribbons (structure is shown in the inset (b) of Figure 5). The $\kappa_{2} \|$ of GB and oxidized GB with full $\mathrm{O}$ coverage as a function of their total lengths are shown in Figure 5 (red circles and black squares respectively). $\kappa_{2}$ becomes saturated quickly with increasing GB length, whether nonoxidized or oxidized. The $\kappa_{2} \|$ values shown in Figure 5 are used as the inputs for $\kappa_{2}$ in Equation (5), and the saturated $\kappa_{2} \|$ values are used as the input $\kappa_{2}$ in Equation (5) for the cases with GBs (oxidized GBs) longer than the cases shown in Figure 5. 
For the oxidized PG with overall $0.5 \%$ and $2.5 \% \mathrm{O}$ coverage (blue and magenta triangles in Figure 4(a)), the $\mathrm{O}$ atoms reside at both graphene GBs and graphene grains. For these two cases, we keep oxidized GB with full $\mathrm{O}$ coverage, and adjust the number of $\mathrm{O}$ atoms in the grains to maintain the same overall $\mathrm{O}$ coverage when the grain size increases. All the $\kappa$ values of the oxidized grains, which are the input $\kappa_{1}$ in Equation (4) and (5) in our EMA model, are extracted from the $\kappa$ values of graphene oxide from Ref. [28]. It is noted that all the error bars of EMA results are obtained by the error propagation method according to the errors of MD simulation results.

\section{Acknowledgement}

This work is funded by the Semiconductor Research Corporation (contract number: 2013-MA2383). This research is supported in part by the Notre Dame Center for Research Computing and NSF through XSEDE resources provided by SDSC Trestles and TACC Lonestar under grant number TG-CTS100078. T.L. also thanks the support from National Science Foundation EFRI2DARA program (1433490).

\section{References}

1. Novoselov, K. S. et al. Electric field effect in atomically thin carbon films. Science 306, 666669 (2004).

2. Novoselov, K. S. et al. Two-dimensional gas of massless Dirac fermions in graphene. Nature 438, 197-200 (2005). 
3. Geim, A. K. \& Novoselov, K. S. The rise of graphene. Nat. Mater. 6, 183-191 (2007).

4. Zhang, Y. B., Tan, Y. W., Stormer, H. L. \& Kim, P. Experimental observation of the quantum Hall effect and Berry's phase in graphene. Nature 438, 201-204 (2005).

5. Balandin, A. A. et al. Superior thermal conductivity of single-layer graphene. Nano Lett. 8 , 902-907 (2008).

6. Cai, W. et al. Thermal transport in suspended and supported monolayer graphene grown by chemical vapor deposition. Nano Lett. 10, 1645-1651 (2010).

7. Chen, S. et al. Raman measurements of thermal transport in suspended monolayer graphene of variable sizes in vacuum and gaseous environments. ACS Nano 5, 321-328 (2011).

8. Lee, J., Yoon, D., Kim, H., Lee, S. W. \& Cheong, H. Thermal conductivity of suspended pristine graphene measured by Raman spectroscopy. Phys. Rev. B 83, 081419(R) (2011).

9. Yan, Z., Liu, G., Khan, J. M. \& Balandin, A. A. Graphene quilts for thermal management of high-power GaN transistors. Nat. Commun. 3, 827 (2012).

10. Jiang, T. et al. Covalent bonding modulated graphene-metal interfacial thermal transport. Nanoscale 8, 10993-11001 (2016).

11. Li, X. S. et al. Large-Area Synthesis of High-Quality and Uniform Graphene Films on Copper Foils. Science 324, 1312-1314 (2009).

12. Mattevi, C., Kim, H. \& Chhowalla, M. A review of chemical vapour deposition of graphene on copper. J. Mater. Chem. 21, 3324-3334 (2011). 
13. Levendorf, M. P., Ruiz-Vargas, C. S., Garg, S. \& Park, J. Transfer-Free Batch Fabrication of Single Layer Graphene Transistors. Nano Lett. 9, 4479-4483 (2009).

14. Ismach, A. et al. Direct Chemical Vapor Deposition of Graphene on Dielectric Surfaces. Nano Lett. 10, 1542-1548 (2010).

15. Bae, S. et al. Roll-to-roll production of 30-inch graphene films for transparent electrodes. Nat. Nanotech. 5, 574-578 (2010).

16. Liu, Y. Y. \& Yakobson, B. I. Cones, Pringles, and Grain Boundary Landscapes in Graphene Topology. Nano Lett. 10, 2178-2183 (2010).

17. Yazyev, O. V. \& Louie, S. G. Topological defects in graphene: Dislocations and grain boundaries. Phys. Rev. B 81, 195420 (2010).

18. Malola, S., Hakkinen, H. \& Koskinen, P. Structural, chemical, and dynamical trends in graphene grain boundaries. Phys. Rev. B 81, 165447 (2010).

19. Grantab, R., Shenoy, V. B. \& Ruoff, R. S. Anomalous strength characteristics of tilt grain boundaries in graphene. Science 330, 946-948 (2010).

20. Huang, P. Y. et al. Grains and grain boundaries in single-layer graphene atomic patchwork quilts. Nature 469, 389-392 (2011).

21. Kim, K. et al. Grain Boundary Mapping in Polycrystalline Graphene. ACS Nano 5, 21422146 (2011). 
22. Song, Z., Artyukhov, V. I., Yakobson, B. I. \& Xu, Z. Pseudo Hall-Petch Strength Reduction in Polycrystalline Graphene. Nano Lett. 13, 1829-1833 (2013).

23. Bagri, A., Kim, S., Ruoff, R. S. \& Shenoy, V. B. Thermal transport across Twin Grain Boundaries in Polycrystalline Graphene from Nonequilibrium Molecular Dynamics Simulations. Nano Lett. 11, 3917-3921 (2011).

24. Lu, Y. \& Guo, J. Thermal transport in grain boundary of graphene by non-equilibrium Green's function approach. Appl. Phys. Lett. 101, 043112 (2012).

25. Aksamija, Z. \& Knezevic, I. Lattice thermal transport in large-area polycrystalline graphene. Phys. Rev. B 90, 035419 (2014).

26. Tahy, K. et al. High field transport properties of 2D and nanoribbon graphene FETs. Device Research Conference, 2009. DRC 2009, 207-208.

27. Lindsay, L., Broido, D. A. \& Mingo, N. Flexural phonons and thermal transport in graphene. Phys. Rev. B 82, 115427 (2010).

28. Mu, X., Wu, X., Zhang, T., Go, D. B. \& Luo, T. Thermal Transport in Graphene Oxide From Ballistic Extreme to Amorphous Limit. Sci. Rep. 4, 3909 (2014).

29. Zhang, H., Fonseca, A. F. \& Cho, K. Tailoring Thermal Transport Property of Graphene through Oxygen Functionalization. J. Phys. Chem. C 118, 1436-1442 (2014).

30. Lin, S. \& Buehler, M. J. Thermal transport in monolayer graphene oxide: Atomistic insights into phonon engineering through surface chemistry. Carbon 77, 351-359 (2014). 
31. Duong, D. L. et al. Probing graphene grain boundaries with optical microscopy. Nature 490, 235-239 (2012).

32. Yan, J. A. \& Chou, M. Y. Oxidation functional groups on graphene: Structural and electronic properties. Phys. Rev. B 82, 125403 (2010).

33. Mu, X., Zhang, T., Go, D. B. \& Luo, T. Coherent and incoherent phonon thermal transport in isotopically modified graphene superlattices. Carbon 83, 208-216 (2015).

34. Mu, X. et al. Ultra-low Thermal Conductivity in Si/Ge Hierarchical Superlattice Nanowire. Sci. Rep. 5, 16697 (2015).

35. Casimir, H. B. G. Note on the Conduction of Heat in Crystals. Physica 5, 495-500 (1938).

36. Luo, T. \& Chen, G. Nanoscale heat transfer - from computation to experiment. Phys. Chem. Chem. Phys. 15, 3389-3412 (2013).

37. Ni, B., Lee, K. H. \& Sinnott, S. B. A reactive empirical bond order (REBO) potential for hydrocarbon-oxygen interactions. J.Phys.: Condens.Matter 16, 7261-7275 (2004).

38. Brenner, D. W. Empirical potential for hydrocarbons for use in simulating the chemical vapor deposition of diamond films. Phys. Rev. B 42, 9458-9471 (1990).

39. Brenner, D. W. et al. A second-generation reactive empirical bond order (REBO) potential energy expression for hydrocarbons. J.Phys.:Condens.Matter 14, 783-802 (2002).

40. Wang, Y., Song, Z. \& Xu, Z. Characterizing phonon thermal conduction in polycrystalline graphene. J. Mater. Res. 29, 362-372 (2014). 
41. Leach, A. G. The thermal conductivity of fomas: 1. Models for heat conduction. J. Phys. D Appl. Phys. 26, $733-739$ (1993).

42. Gong, L., Wang, Y., Cheng, X., Zhang, R. \& Zhang, H. A novel effective medium theory for modelling the thermal conductivity of porous materials. Int. J. Heat and Mass Tran. 68, 295-298 (2014).

43. Plimpton, S. Fast parallel algorithms for short-range molecular dynamics. J.Comput.Phys. 117, 1-19 (1995).

44. Song, Z., Mu, X., Luo, T. \& Xu, Z. Unzipping of carbon nanotubes is geometry-dependent. Nanotechnology 27, 015601 (2016).

45. Arfken, G. in Mathematical methods for physicists 428-436 (Orlando, FL: Academic Press, 1985).

46. Nose, S. A unified formulation of the constant temperature molecular dynamics methods. J.Chem.Phys. 81, 511-519 (1984).

47. Hoover, W. G. Canonical dynamics: Equilibrium phase-space distributions. Phys. Rev. A 31, 1695-1697 (1985).

48. Schneider, T. \& Stoll, E. Molecular-dynamics study of a three-dimensional one-component model for distortive phase transitions. Phys. Rev. B 17, 1302-1322 (1978).

49. Luo, T., Esfarjani, K., Shiomi, J., Henry, A. \& Chen, G. Molecular dynamics simulation of thermal energy transport in polydimethylsiloxane. J. Appl. Phys. 109, 074321 (2011). 
50. Kundu, A. et al. Heat conduction and phonon localization in disordered harmonic crystals.

Europhys. Lett. 90, 40001 (2010). 